\title{
Síndrome Metabólica, Seus Fenótipos e Resistência à Insulina pelo HOMA-RI
}

\section{artigo original}

ERNESTO P. DE OLIVEIRA

MARIA DAS DORES A. DE LIMA Mirabeau leVI A. DE SOUZA

Faculdades de Farmácia (EPO \& MLAS) e de Medicina (MDAL), Universidade Federal da Bahia, Salvador, BA.
Recebido em 02/04/07 Aceito em 20/07/07

\section{RESUMO}

O diagnóstico de síndrome metabólica (SM) segundo o National Cholesterol Education Program Adult Treatment Panel III não reflete necessariamente a presença de resistência à insulina (RI), um potencial alvo terapêutico para prevenção de diabetes tipo 2 e doenças cardiovasculares. Em estudo de corte transversal, assentado em dados anteriores de prevalência, avaliamos o comportamento do HOMA-RI, um parâmetro de $\mathrm{RI}$ bem difundido, frente à $\mathrm{SM}$ e anormalidades associadas. HOMA-RI foi maior nos indivíduos com SM $(2,8 \pm 1,6$ vs. $1,8 \pm 1,4)(p<$ $0,001)$ e mostrou excelente correlação com insulinemia de jejum $\left(r_{S}=\right.$ $0,961)$. HOMA-RI $\geq 2,5$ aliou bons níveis de especificidade e sensibilidade para a associação de SM e RI. Diferente de aumento da glicemia, obesidade abdominal e elevação da trigliceridemia, componentes da SM mais bem relacionados com $\mathrm{RI}$, a elevação da pressão arterial e a redução do HDL-c não mostraram associação com $\mathrm{HOMA}-\mathrm{RI} \geq 2,5$. A demonstração de que alguns fenótipos de SM ou anormalidades associadas foram mais preditivos de RI pode apontar para a possibilidade de uso do índice como um indicador de RI associada à SM. (Arq Bras Endocrinol Metab 2007;51/9:1506-1515)

Descritores: Resistência à insulina; Síndrome $\mathrm{X}$ metabólica; Diagnóstico; HOMA (homeostatic model assessment)

\begin{abstract}
Metabolic Syndrome, Its Phenotypes, and Insulin Resistance by HOMA-IR.

The diagnosis of the metabolic syndrome (MS) according to the National Cholesterol Education Program Adult Treatment Panel III does not reflect necessarily the presence of insulin resistance (IR), a potential therapeutical target for type 2 diabetes and cardiovascular disease prevention. Based on previous prevalence data, a cross-sectional study was conducted to determine the HOMA-IR relationship to the MS and some associated abnormalities. HOMA-IR was higher in individuals with the MS $(2.8 \pm 1.6$ vs. $1.8 \pm$ 1.4) $(p<0.001)$. HOMA-IR $\geq 2.5$ allied good specificity and sensitivity levels for the association of MS and IR. Hyperglycemia, hypertrigliceridemia, and abdominal obesity, the MS components best related to IR, were statistically associated with HOMA-IR $\geq 2.5$, but not hypertension neither low HDL-c. The demonstration that some of MS phenotypes or associated abnormalities were more predictive for IR could point out to the possibility of the use of the index as a marker of the presence of IR associated to MS. (Arq Bras Endocrinol Metab 2007;51/9:1506-1515)
\end{abstract}

Keywords: Insulin resistance; Metabolic syndrome X; Diagnosis; HOMA (homeostatic model assessment) 
$\mathrm{E}$ MBORA A ASSOCIAÇÃO ENTRE resistência à insulina (RI) e risco de doenças cardiovasculares (DCV) seja referida desde o final da década de 60 (1), a partir das publicações de Reaven (1988) e Kaplan (1989) o tema ganhou maior amplitude na literatura. Em seus artigos, os autores argumentam sobre a coexistência freqüente de fatores de risco para DCV em um mesmo indivíduo, quais sejam: redução da tolerância à glicose, dislipidemia e hipertensão, postulam a RI como um nexo causal entre eles, põem a obesidade como amplificadora da redução da sensibilidade tissular ao hormônio e propõem a existência de uma síndrome metabólica (SM), denominada de "síndrome X", por Reaven (2), e de "Quarteto Mortal”, por Kaplan (3).

Uma das definições de SM mais freqüentemente utilizadas é proposta pelo National Cholesterol Education Program Adult Treatment Panel III (4) e está baseada na presença de 3 ou mais componentes da SM em um mesmo indivíduo: obesidade abdominal, redução da tolerância à glicose, elevação da trigliceridemia, diminuição dos níveis de colesterol HDL e aumento da pressão arterial. $\mathrm{O}$ atendimento ao requisito da definição não reflete necessariamente a presença de RI, um potencial alvo terapêutico para prevenção de diabetes tipo 2 e doenças cardiovasculares.

A preocupação com o envolvimento da RI na associação de fatores de risco cardiovascular se reforça pelos comentários de Stern e cols. (5), bem como pela postura mais recente do Colégio Americano de Endocrinologia (ACE) (6), que refere triglicérides elevado, HDL-c reduzido, pressão arterial aumentada e glicemia de jejum ou pós-prandial elevada como "anormalidades identificadoras" de possíveis portadores de RI. Quanto maior o número e a intensidade das anormalidades, maior a probabilidade de o indivíduo ser insulino-resistente.

Um dos parâmetros de medida de RI mais difundidos, sobretudo por sua simplicidade operacional, é o índice HOMA-RI (homeostatic model assessment - insulin resistance), descrito por Matthews e cols. (7) e validado por diversos autores para estudos epidemiológicos, mas ainda não convenientemente recomendado para uso na prática clínica (8-11).

A partir dos dados de um estudo de prevalência de SM (12), avaliamos o comportamento dos valores do índice HOMA-RI frente à síndrome e anormalidades associadas, com o intuito de determinar a possível associação de RI com SM, bem como os valores preditivos das suas alterações isoladas sobre aumento do índice.

\section{MATERIAL E MÉTODOS}

O modelo de corte transversal foi aplicado sobre a amostra de um estudo de prevalência de SM, de base populacional, realizado no distrito de Cavunge, Município de Ipecaetá, semi-árido baiano. Naquele estudo, com base na prevalência estimada de diabetes, considerou-se uma amostra aleatória simples de 270 indivíduos (12).

Os critérios de elegibilidade dos sujeitos da pesquisa, que já estão referidos no trabalho anterior, foram acrescidos da exclusão daqueles que declararam ter diabetes.

Também, as variáveis definidas, métodos empregados e critérios de diagnóstico foram descritos naquele artigo, exceto a determinação da insulinemia de jejum e o cálculo do índice HOMA-RI.

A insulinemia de jejum (INS) foi medida por ELISA. O método da Linco Research ${ }^{\circledR}$ (St. Charles, MO - USA) é do tipo sanduíche, que utiliza dois anticorpos monoclonais anti-insulina humana, não havendo reação cruzada com a pró-insulina e com seu principal metabólito, a des-31,32pró-insulina humana, o que se constitui interferente importante na utilização do HOMA no diagnóstico de RI. O sistema ELISA Linco Research ${ }^{\circledR}$ guarda excelente correlação com radioimunoensaio também específico para insulina humana $(\mathrm{r}=0,978)$, conforme o fabricante dos dois kits. Ainda conforme dados do fabricante, a sensibilidade analítica é de $2 \mu \mathrm{U} / \mathrm{mL}$, reação cruzada com pró-insulina não é detectada até a concentração de $100 \mathrm{nM} / \mathrm{L}$, bem acima dos $0,02 \mathrm{nM} / \mathrm{L}$ que são alcançados no momento pósprandial (13), e sua variabilidade analítica situa-se em torno de 6 e $10 \%$; em nossa experiência, foi de $9,1 \%$.

$\mathrm{O}$ índice HOMA-RI foi calculado como segue: glicemia de jejum $(\mathrm{mmol} / \mathrm{L}) \mathrm{x}$ insulinemia de jejum $(\mathrm{mU} / \mathrm{L}) / 22,5$.

As análises estatísticas foram realizadas com ajuda do software SPSS 9.0 e, para efeito de interpretação, o limite de erro tipo I foi de até $5 \%(\mathrm{p} \leq 0,05)$.

Os valores de HOMA-RI, uma vez que mostraram distribuição não paramétrica, mesmo após transformação logarítmica, foram comparados através do Mann-Whitney; variáveis ordinais e nominais foram comparadas pelo teste do qui-quadrado $\left(\chi^{2}\right)$; o coeficiente de correlação de Spearman $\left(r_{S}\right)$ foi utilizado para estabelecer correlação entre variáveis ordinais e contínuas; o índice HOMA-RI foi avaliado através de curva ROC (receiver-operating characteristic) para definição de valores de corte que maximizassem a sensibilidade e especificidade diagnóstica para a associação de SM com RI. Razão de prevalência com intervalo de confiança de 95\% (RP; IC 95\%) foi empregada como medida de efeito para evitar super-estimativas produzidas pelo uso de Razão de Chances.

O estudo foi autorizado após avaliação do Comitê de Ética em Pesquisa Envolvendo Seres Humanos da Universidade Estadual de Feira de Santana (CEP/ UEFS), conforme resolução do Conselho Nacional de Saúde número 196, de 10 de outubro de 1996. 


\section{RESULTADOS}

A amostra final foi constituída por 227 sujeitos (fração amostral $=48,6 \%)$, após subtraídos $24(8,9 \%)$ indivíduos que não atenderam ao convite ou não foram encontrados, $14(5,2 \%)$ exclusões por aplicação dos critérios definidos, das quais $8(3,0 \%)$ portadores de diabetes pré-estabelecido, e $5(1,8 \%)$ casos com insulinemia abaixo da sensibilidade analítica do teste.

Características gerais da amostra estudada, inclusive as prevalências das alterações metabólicas envolvidas com a SM, são apresentadas na tabela 1 .

Mesmo após tendo sido excluídos alguns indivíduos, em relação ao trabalho anterior, a prevalência de SM continuou sendo de 30,0\% (68 indivíduos), tendo sido mais elevada em indivíduos com 45 ou mais anos de idade $(\mathrm{p}<0,001)$ e maior entre as mulheres $(\mathrm{p}=0,002)$. As frequiências de redução de HDL-c e de elevação de PA foram bem maiores que as taxas dos outros três componentes, sobretudo entre os homens. Entre as mulheres, a ocorrência de obesidade abdominal também se destacou (12).

A distribuição dos valores de HOMA-RI mostrou excelente correlação com os níveis de insulinemia de jejum $\left(\mathrm{r}_{\mathrm{S}}=0,961\right)$.

HOMA-RI foi igual a $2,1 \pm 1,5$, com mediana de 1,7. O valor do índice foi de $2,3 \pm 1,6$, para indivíduos do sexo feminino, maior que o resultado de $1,9 \pm 1,3$, encontrado nos indivíduos do sexo masculino $(\mathrm{p}=0,023)$. Os níveis de HOMA-RI não se correlacionaram significativamente com o crescimento da idade $\left(r_{S}=0,043\right)$ e não se mostraram significativamente diferentes entre as diversas faixas etárias: 25 a 34 anos, 35 a 44 anos, 45 a 54 anos, 55 a 64 anos e 65 ou mais anos de idade.

HOMA-RI diferiu entre indivíduos com e sem SM, respectivamente: $2,8 \pm 1,6$ e $1,8 \pm 1,4(p<$ $0,001)$. Também, conforme os critérios do NCEP-

Tabela 1. Características gerais da amostra estudada. Cavunge, 2005.

\begin{tabular}{|c|c|}
\hline CARACTERÍSTICA & Valor extraído da amostra de 227 indivíduos ${ }^{1}$ \\
\hline Sexo Masculino & $43,2 \%$ (98 indivíduos) \\
\hline Sexo Feminino & $56,8 \%$ (129 indivíduos) \\
\hline Idade & $49,0 \pm 15,0$ anos (variando de 25 a 87 anos) \\
\hline Peso (kg) & $65,4 \pm 12,2$ \\
\hline Altura (m) & $1,59 \pm 0,09$ \\
\hline $\mathrm{IMC}\left(\mathrm{kg} / \mathrm{m}^{2}\right)$ & $25,7 \pm 4,1$ \\
\hline Excesso de peso grau I & $41,0 \%$ (93 indivíduos) \\
\hline Excesso de peso grau lla & $11,5 \%$ (26 indivíduos) \\
\hline Excesso de peso grau Ilb & 3,1\%(7 indivíduos) \\
\hline Diâmetro da cintura (DC) & $88,1 \pm 10,5$ \\
\hline DC aumentado 2 & $22,9 \%$ (52 indivíduos) \\
\hline DC significativamente aumentado 3 & 31,3\% (71 indivíduos) \\
\hline PAS $(\mathrm{mmHg})$ & $130,1 \pm 23,3(120,0)$ \\
\hline PAD $(\mathrm{mmHg})$ & $82,3 \pm 11,7(80,0)$ \\
\hline Hipertensão arterial & 43,6\% (99 indivíduos) \\
\hline Glicemia (mg/dL) & $96,2 \pm 22,3(92,0)$ \\
\hline Glicose de jejum prejudicada & $18,9 \%$ (43 indivíduos) \\
\hline Diabetes & $6,2 \%$ (14 indivíduos) \\
\hline Insulinemia de jejum (mU/L) & $8,6 \pm 5,1(7,3)$ \\
\hline HOMA-RI & $2,1 \pm 1,5(1,7)$ \\
\hline Colesterol total (mg/dL) & $210,0 \pm 47,4(208,0)$ \\
\hline Colesterolemia $\geq 240 \mathrm{mg} / \mathrm{dL}$ & 23,3\% (53 indivíduos) \\
\hline Triglicérides (mg/dL) & $112,9 \pm 83,2(95,0)$ \\
\hline Trigliceridemia de jejum > 200 mg/dL & $7,9 \%$ (18 indivíduos) \\
\hline HDL-colesterol (mg/dL) & $40,3 \pm 10,1(38,0)$ \\
\hline Concentração de HDL-c $<40$ mg/dL & $56,8 \%$ (129 indivíduos) \\
\hline Síndrome metabólica & $30,0 \%$ (68 indivíduos) \\
\hline
\end{tabular}


ATPIII (4), o índice foi significativamente maior entre os indivíduos com obesidade abdominal, elevação da trigliceridemia e aumento da glicemia. Os valores de HOMA-RI não diferiram entre indivíduos com e sem redução do HDL-c ou entre aqueles com e sem elevação da pressão arterial (tabela 2). Especificamente quanto à presença ou ausência de obesidade abdominal, a diferença do HOMA-RI não se revelou estatisticamente significante entre os homens $(2,4 \pm$ $1,3$ vs. $1,8 \pm 1,3 / \mathrm{p}=0,084)$, somente nas mulheres $(2,67 \pm 1,66$ vs. $1,92 \pm 1,52 / p=0,002)$.

Houve uma tendência de crescimento $(\mathrm{p}<$ $0,001)$ do índice HOMA-RI frente à agregação de componentes da SM $\left(r_{S}=0,334\right)$, a qual foi mais forte entre as mulheres $\left(r_{S}=0,401\right)$. Os valores de HOMARI também mostraram alguma correlação com a amplitude de algumas das alterações relacionadas, quais sejam: níveis de obesidade $\left(\mathrm{r}_{\mathrm{S}}=0,344\right)$ e de obesidade abdominal $\left(r_{S}=0,273\right)$; estado de tolerância à glicose $\left(r_{S}=0,315\right)$ e taxa de glicemia $\left(r_{S}=0,403\right)$; concentração de triglicérides $\left(r_{S}=0,217\right)$. $O$ índice não mostrou correlação significativa com a diminuição da concentração de HDL-c $\left(r_{S}=-0,057 ; p=0,396\right)$ ou com os graus de hipertensão arterial $\left(\mathrm{r}_{\mathrm{S}}=0,033 ; \mathrm{p}\right.$ $=0,676$ ) (figura 1 ).

A área sob a curva ROC (receiver-operating characteristic) do HOMA-RI em relação à presença de SM (4), como diagnóstico presuntivo de RI, foi de $0,714(\mathrm{p}<0,001)$ (figura 2). Como esperado, maiores valores de corte do HOMA-RI produziram aumento da especificidade diagnóstica da associação de SM com
RI e do valor preditivo positivo da elevação do índice, com redução da sensibilidade e do valor preditivo negativo.

O valor de HOMA-RI igual ou maior que 2,5 associou bom nível de especificidade $(83,6 \%)$ com sensibilidade de $47,1 \%$, valores preditivos negativo de $78,7 \%$ e positivo de $55,2 \%$ (tabela 3 ); além de ser mais próximo de 2,3 , resultado equivalente ao quartil superior das insulinemias de jejum dos não diabéticos, que constitui condição indispensável ao diagnóstico de síndrome de resistência à insulina, segundo critérios do EGIR - European Group for the Study of Insulin Resistance (14). A freqüência de indivíduos com HOMA-RI $\geq 2,5$ foi de $25,6 \%$, pouco menor que a prevalência de SM de 30,0\%.

A definição de uma zona cinza onde a razão de verossimilhança foi de 1,0 , situada entre dois pontos definidos, HOMA-RI abaixo de 1,5, privilegiando a sensibilidade e a segurança em excluir a associação de $\mathrm{SM}$ com RI, e igual ou maior que 2,5 , com maior especificidade para ela (tabela 3 ), permitiu estabelecer uma escala de freqüência da coexistência de SM e níveis de HOMA-RI. Em relação aos indivíduos com HOMA-RI abaixo de 1,5 , a associação com SM foi 2,2 vezes mais freqüente quando o valor HOMA-RI ficou na zona cinza, 4,0 vezes quando HOMA-RI foi igual ou maior que 2,5, e 4,4 vezes quando HOMA-RI foi maior ou igual a 3,5 .

RI definida por HOMA-RI $\geq 2,5$ esteve presente em $34,1 \%$ dos indivíduos obesos ou com sobrepeso $(\mathrm{RP}=2,39 ; 1,39-4,10) ; 37,2 \%$ dos sujeitos

Tabela 2. Valores de HOMA-RI na presença e ausência de condições relacionadas à síndrome metabólica, conforme critérios do National Cholesterol Education Program Adult Treatment Panel III (4). Cavunge, 2005.

\begin{tabular}{lccc}
\hline CONDIÇÃO & $\begin{array}{c}\text { HOMA-RI NA } \\
\text { PRESENÇA (a) }\end{array}$ & $\begin{array}{c}\text { HOMA-RI NA } \\
\text { AUSÊNCIA (b) }\end{array}$ & $\begin{array}{c}\text { SIGNIFICÂNCIA } \\
\text { ESTATísTICA } \\
\text { (a-b) }\end{array}$ \\
Síndrome metabólica & $2,8 \pm 1,6$ & $1,8 \pm 1,4$ & $\mathrm{p}<0,001$ \\
$\downarrow$ HDL-c & $2,2 \pm 1,6$ & $1,9 \pm 1,2$ & $\mathrm{p}=0,205$ \\
$\uparrow \mathrm{PA}$ & $2,1 \pm 1,3$ & $2,1 \pm 1,7$ & $\mathrm{p}=0,178$ \\
$\mathrm{OA}$ & $2,6 \pm 1,6$ & $1,9 \pm 1,4$ & $\mathrm{p}<0,001$ \\
$\uparrow T \mathrm{Tg}$ & $2,6 \pm 1,6$ & $2,0 \pm 1,5$ & $\mathrm{p}=0,001$ \\
$\uparrow \mathrm{GLI}$ & $3,6 \pm 2,3$ & $1,9 \pm 1,2$ & $\mathrm{p}<0,001$ \\
\hline
\end{tabular}

$\mathrm{SM}=$ Síndrome metabólica, $\downarrow \mathrm{HDL}-\mathrm{c}=$ redução do $\mathrm{HDL}-\mathrm{c}(<40 \mathrm{mg} / \mathrm{dL}$ para homens $\mathrm{e}<50$ para mulheres, $\uparrow P A=$ elevação da pressão arterial (PAS $\geq 130 \mathrm{mmHg}$ e/ou $P A D \geq 85$, ou uso de anti-hipertensivo), $\mathrm{OA}=$ obesidade abdominal $(>102 \mathrm{~cm}$ para homens e $>88$ para mulheres), $\uparrow \mathrm{Tg}=$ elevação da trigliceridemia ( $\geq 150 \mathrm{mg} / \mathrm{dL}$ ), $\uparrow \mathrm{GLI}=$ aumento da glicemia ( $\geq$ $110 \mathrm{mg} / \mathrm{dL}$ ou tratamento).

1 Teste de Mann-Whitney. 

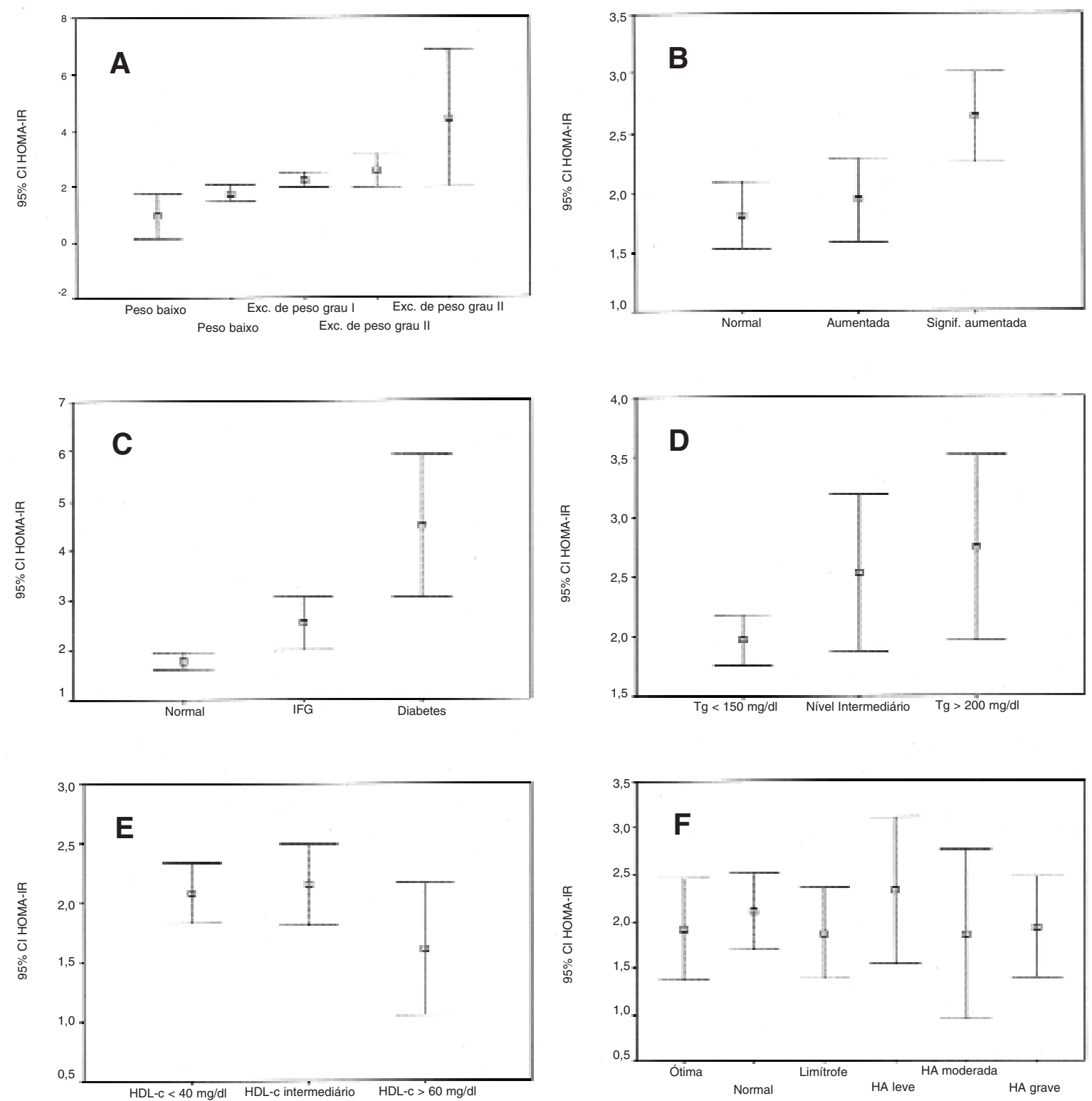

Figura 1. Valores de HOMA-IR em relação a: A) classificação de obesidade (OMS, 1997), B) classificação de obesidade abdominal (OMS, 1997), C) estados da tolerância à glicose (ADA, 2004), D) patamares de trigliceridemia (SBC, 2001), E) níveis de HDL-c (SBC, 2001) e F) estados da pressão arterial (SBH, 2002). 


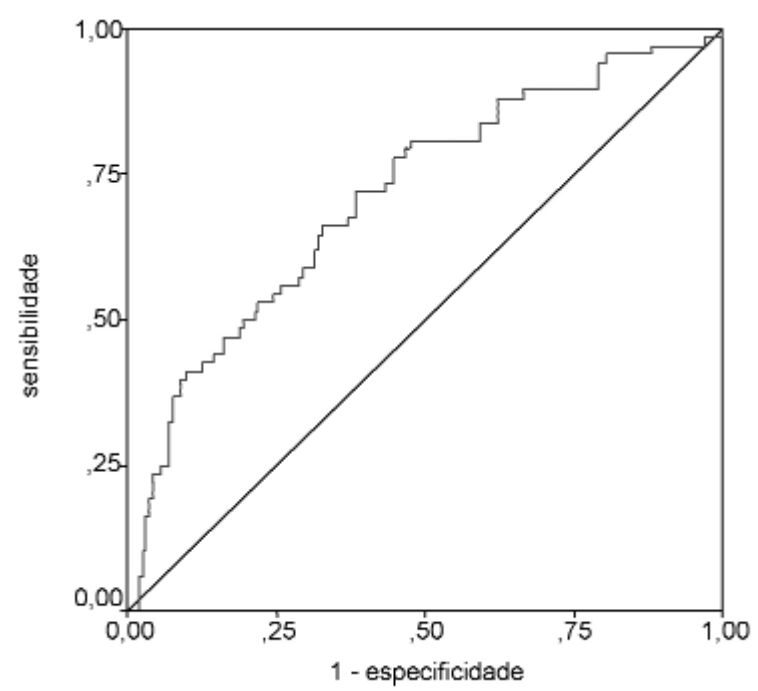

Figura 2. Curva ROC com SM (4) como estado real de positividade.

Tabela 3. Propriedades do HOMA-RI enquanto teste de diagnóstico segundo alguns valores de corte propostos.

\begin{tabular}{lllllll}
\hline $\begin{array}{l}\text { VALOR DE } \\
\text { CORTE }\end{array}$ & SD & ED & VPP & VPN & RV & EfT \\
\hline HOMA-RI $\geq 1,5$ & $80,9 \%$ & $50,9 \%$ & $41,3 \%$ & $86,2 \%$ & 1,6 & $59,9 \%$ \\
HOMA-RI $\geq 2,0$ & $57,3 \%$ & $70,4 \%$ & $45,3 \%$ & $79,4 \%$ & 1,9 & $66,5 \%$ \\
HOMA-RI $\geq 2,5$ & $47,1 \%$ & $83,6 \%$ & $55,2 \%$ & $78,7 \%$ & 2,9 & $72,7 \%$ \\
\hline HOMA-RI $\geq 3,0$ & $41,1 \%$ & $88,7 \%$ & $60,9 \%$ & $77,9 \%$ & 3,7 & $74,4 \%$ \\
\hline
\end{tabular}

SD = Sensibilidade diagnóstica, ED = Especificidade diagnóstica, VPP = Valor preditivo do teste positivo, VPN = Valor preditivo do teste negativo, RV = Razão de verossimilhança, EfT = Eficiência do teste (área sob a curva ROC).

com IFG $(R P=1,63 ; 1,02-2,61)$ e $78,6 \%$ dos diabéticos $(\mathrm{RP}=3,56 ; 2,45-5,17) ;$ em $55,6 \%$ dos hipertrigliceridêmicos $(\mathrm{RP}=2,42 ; 1,49-3,92) ; 27,3 \%$ dos hipertensos $(\mathrm{RP}=1,13 ; 0,72-1,76)$ e em $24,0 \%$ dos indivíduos com HDL-c abaixo de $40 \mathrm{mg} / \mathrm{dL}$ (RP $=0,87 ; 0,56-1,36)$. HOMA $\geq 2,5$ também esteve presente em $14,3 \%$ dos indivíduos com peso normal $(\mathrm{RP}=0,42 ; 0,24-0,72)$, em $18,5 \%$ daqueles com NGT $(R P=0,40 ; 0,26-0,62)$, em $23,0 \%$ dos normotrigliceridêmicos $(\mathrm{RP}=0,41 ; 0,26-0,67)$, $24,2 \%$ dos normotensos $(\mathrm{RP}=0,89 ; 0,57-1,38)$; $11,1 \%$ dos indivíduos com HDL-c acima de 60 $\mathrm{mg} / \mathrm{dL}(\mathrm{RP}=0,42 ; 0,07-2,73)$ e $27,6 \%$ daqueles com HDL-c $>40 \mathrm{mg} / \mathrm{dL}(\mathrm{RP}=1,15 ; 0,74-1,79)$.

HOMA-RI $\geq 2,5$ classificou como resistentes à insulina $47,1 \%$ dos sujeitos com $\mathrm{SM}(\mathrm{RP}=2,59$; $1,79-3,75), 81,8 \%$ dos indivíduos com SM que tinham OA e aumento da glicemia entre os compo- nentes presentes da síndrome $(\mathrm{RP}=3,61 ; 2,49-5,23)$, $70,0 \%$ dos indivíduos com SM que apresentavam elevação da trigliceridemia e aumento da glicemia (RP $=2,98 ; 1,86-4,77), 64,7 \%$ daqueles com SM e elevação de trigliceridemia mais $\mathrm{OA}(\mathrm{RP}=2,89$; $1,88-4,45)$, e $30,6 \%$ dos casos de SM em que somente um desses três componentes mais especificamente relacionados à RI estava presente, associado à elevação da pressão arterial e redução do HDL-c $(\mathrm{RP}=1,24$; $0,72-2,16)$. De todos os indivíduos analisados, HOMA-RI $\geq 2,5$ incluiu $16,4 \%$ dos sujeitos sem SM; $15,6 \%$ daqueles que tinham não mais que um dos componentes da síndrome e $11,7 \%$ dos que não apresentavam qualquer um dos três componentes mais concretamente associados à RI, quais sejam: aumento da glicemia, OA e elevação da trigliceridemia (tabela 4).

SM esteve presente em $13,8 \%$ daqueles com HOMA-RI $<1,5(\mathrm{RP}=0,33 ; 0,19-0,58)$ e em $55,2 \%$ 
Tabela 4. VP+ de alguns fenótipos relacionados à síndrome metabólica para RI definida por HOMA-RI $\geq 2,5$.

\begin{tabular}{lc}
\hline Fenótipo & VP+ (\%) para RI \\
SM com OA e aumento da glicemia & 81,8 \\
Diabetes & 78,6 \\
SM com elevação da trigliceridemia e aumento da glicemia & 70,0 \\
SM com elevação da trigliceridemia e OA & 64,7 \\
Hipertrigliceridemia & 55,6 \\
SM & 47,1 \\
IFG & 37,2 \\
Sobrepeso ou obesidade & 34,1 \\
SM com elevação da trigliceridemia ou aumento da glicemia ou OA & 30,6 \\
Hipertensão & 27,3 \\
HDL-c < 40 mg/dL & 24,0 \\
\hline
\end{tabular}

$\mathrm{VP}+=$ Valores preditivos positivos, $\mathrm{RI}=$ resistência à insulina, HOMA-RI = homeostasis assessment model - resistência à insulina, $\mathrm{SM}=$ síndrome metabólica, $\mathrm{OA}=$ obesidade abdominal, $\mathrm{IFG}=$ glicemia de jejum prejudicada, HDL-c = colesterol da HDL.

daqueles com HOMA-RI $\geq 2,5 \quad(\mathrm{RP}=2,59$; 1,79-3,75). Nesses últimos, foi mais freqüente quando constituída também por OA e aumento da glicemia $(R P=13,03 ; 2,90-58,58)$; elevação da trigliceridemia e aumento da glicemia $(\mathrm{RP}=6,80$; $1,82-25,43)$; elevação de trigliceridemia mais OA (RP $=5,34 ; 2,07-13,80)$. Por outro lado, os casos de SM em que somente um desses três componentes mais especificamente relacionados à RI estava presente não foram significativamente mais freqüentes quando HOMA-RI $\geq 2,5(\mathrm{RP}=1,28 ; 0,67-2,44)$.

\section{DISCUSSÃO}

SM pode ser considerada de duas maneiras diferentes: como a agregação de fatores de risco cardiovascular em um mesmo indivíduo, atendendo a um escore de diagnóstico que compõe a definição clínica da própria síndrome, a qual não necessariamente requer o estabelecimento da relação direta com a RI, ou como o conjunto de doenças ou de alterações metabólicas e hemodinâmicas referido à presença de RI e/ou de sua hiperinsulinemia reativa $(15,16)$.

Quando comparamos sujeitos com e sem SM, tal como definida pelo NCEP-ATPIII (4), que não requer medida direta da RI, os valores de HOMA-RI foram significativamente maiores entre os indivíduos portadores da síndrome, achado também referido por outros autores (17-20).

Os valores de HOMA-RI também cresceram à medida da evolução de zero a cinco componentes da
$\mathrm{SM}\left(\mathrm{r}_{\mathrm{S}}=0,334\right)$, concordando com o relato de Saely e cols. (20), com um salto de maior significância entre indivíduos com dois e com três componentes isolados $(\mathrm{p}=0,023)$.

Esse padrão de comportamento do HOMA-RI foi semelhante nos sexos feminino e masculino, embora sua média tenha sido mais elevada entre as mulheres $(\mathrm{p}=$ 0,023 ), grupo em que a prevalência de SM foi significativamente maior, dados também referidos por Resnick e cols. (19) e Tipene-Leach e cols. (21).

Por outro lado, os valores de HOMA-RI não guardaram correlação com o crescimento da idade e não diferiram entre as faixas etárias definidas, divergindo do que se verificou com a prevalência de SM (12).

Os valores de HOMA-RI isoladamente não puderam distinguir claramente indivíduos com ou sem síndrome, apesar da diferença estatística existente entre os dois grupos. Insuficiência também foi aventada por McAuley e cols. (22), Bustos e cols. (23) e Jaber e cols. (24). Aparentemente, isso foi conseqüência da grande dispersão encontrada, que oscilou de um coeficiente de variação $(\mathrm{CV})$ de $57,1 \%$, em indivíduos com SM, a 77,8\%, em sujeitos sem SM.

Dispersões elevadas, entre $50,0 \%$ e $90,6 \%$, são referidas em vários trabalhos publicados, envolvendo uma diversidade de métodos, alguns deles com número amostral bem maior que o nosso (20,25-27).

A coleta de amostra única, em não atendendo às flutuações de insulinemia inerentes ao ciclo de secreção do hormônio $(7,10)$, pode ter contribuído para a dispersão dos resultados. Contudo, tal questão exige análise mais aprofundada, uma vez que a grande 
maioria dos trabalhos publicados refere dosagem de insulina em amostra única e, além disso, a exigência de coletar 3 amostras, uma a cada 5 minutos, para cobrir o ciclo de secreção da insulina, vai de encontro a um dos maiores atrativos para o uso do HOMA-RI, a simplicidade operacional.

Se remontamos à diversidade fenotípica da síndrome e das anormalidades associadas à RI, a própria heterogeneidade da amostra populacional estudada contribuiu para a amplitude das dispersões encontradas.

Da mesma forma, o diagnóstico de síndrome (4) definido como condição de positividade real no traçado da curva ROC pode ter favorecido a insuficiência de o índice distinguir isoladamente presença e ausência de SM, uma vez que tal diagnóstico engloba uma diversidade de combinações de alterações metabólicas e hemodinâmica envolvidas, bem como dos seus graus ou intensidades para o atendimento à definição da síndrome, além de não assegurar a existência de RI associada (28).

Tais diversidades possivelmente resultam na insuficiência do diagnóstico de SM refletir presença de RI. Claramente, isso se ilustra pela observação de que apenas cerca de $50 \%$ dos indivíduos com pressão arterial elevada têm RI, apesar das evidências de uma relação causal entre elas (29).

A participação da redução de HDL-c no diagnóstico da SM também pode favorecer sua desvinculação com a RI, uma vez que a redução de HDL-c com ausência de aumento do $\mathrm{Tg}$ não se associa com hiperinsulinemia nem com características da síndrome de resistência à insulina $(20,30,31)$.

Entre nossos resultados, chama a atenção que as prevalências isoladas de elevação da pressão arterial $(56,4 \%)$ e de redução do HDL-c $(71,8 \%)$ destoam das taxas dos outros três componentes mais bem correlacionados com a RI, cujas freqüências são mais convergentes: de $13,2 \%$ a $31,3 \%$ e, especificamente entre os homens, de $11,2 \%$ a $21,4 \%$. Tais dados são concordantes com os achados de Park e cols. (32) em população negra, bem como com os comentários de Natali \& Ferrannini (33) sobre a possível inespecificidade incorporada ao diagnóstico da SM pelos valores de corte mais sensíveis para elevação da pressão arterial.

Nesse sentido, nossos dados parecem concordar com as potenciais inespecificidades da elevação da pressão arterial e da redução do HDL-c, já que não se demonstraram diferenças significativas dos níveis de HOMA-RI entre indivíduos com e sem elevação da pressão arterial $(\mathrm{p}=0,178)$, tal como relataram Jaber e cols. (24) e Mohteshamzadeh e cols. (34), ou entre sujeitos com e sem redução do HDL-c $(\mathrm{p}=0,205)$, semelhantemente a Taniguchi e cols. (35), Jaber e cols. (24) e Jeffs e cols. (31), que encontraram associação mais fraca com HDL-c. $\mathrm{O}$ índice não mostrou correlação significativa $(p \geq 0,198)$ com a concentração de HDL-c ou com os graus de hipertensão arterial. Hipertensão arterial $(\mathrm{RP}=1,13 ; 0,72-1,76)$ ou HDL-c abaixo de 40 $\mathrm{mg} / \mathrm{dL}(\mathrm{RP}=0,87 ; 0,56-1,36)$ isoladamente não estiveram relacionados com HOMA-RI $\geq 2,5$, o que ocorreu com as demais alterações metabólicas analisadas. Além disso, a freqüência de casos de SM em que somente um dos três componentes mais especificamente relacionados à $\mathrm{RI}$ estava presente, quais sejam: aumento da glicemia, OA e elevação da trigliceridemia, não foi significativamente maior quando HOMA-RI $\geq 2,5$ (RP $=1,28 ; 0,67-2,44)$, o que aconteceu nas demais combinações fenotípicas da síndrome, em que pelo menos dois deles apareciam.

Se, em estudo anterior (12), tais alterações metabólicas isoladas, aparentemente mais específicas de RI, predisseram SM com força discretamente maior que a elevação da pressão arterial ou a redução do HDL-c, neste elas foram mais intensamente associadas com RI definida por HOMA-RI $\geq 2,5$.

Além disso, a variabilidade dos valores de HOMA-RI em relação à diversidade fenotípica da SM pode ser atribuída à maior especificidade do índice para revelar a sensibilidade hepática à insulina, subestimando a participação dos tecidos periféricos (36), o que talvez possa justificar o achado de que nem todos os diabéticos $(78,6 \%)$ foram resistentes à insulina segundo o valor de HOMA-RI (tabela 4).

Tais questões justificam não termos encontrado um valor de corte do HOMA-RI que definisse a associação de SM e RI com eficiência maior que $72,7 \%$, para HOMA-RI $\geq 2,5$, ou 74,4\%, para HOMA-RI $\geq 3,0$, o que significa $27,3 \%$ de resultados supostamente falsos, $15,9 \%$ de falso-negativos e 11,4 de falso-positivos, especificamente para o valor de 2,5 . Para HOMA-RI $\geq$ 3,0 , foram $17,7 \%$ e $7,9 \%$, respectivamente.

Ainda assim, considerando que as distribuições dos resultados de HOMA-RI e de insulinemia de jejum guardaram excelente correlação $\left(\mathrm{r}_{\mathrm{S}}=0,961\right)$, o uso do valor de HOMA-RI $\geq 2,5$ foi preferido por atender à condição de diagnóstico de síndrome de resistência à insulina do EGIR (14).

Os resultados apontam para uma dissociação parcial entre SM e RI definida pelo HOMA-RI, a qual, em grande parte, atribuiu-se às maiores inespecificidades da elevação da pressão arterial e da redução do HDL-c em relação à resistência ao hormônio. Por outro lado, a revelação de um crescimento da freqüência de SM à medida da elevação dos valores de 
HOMA-RI, sobretudo de SM associada a componentes mais especificamente relacionados à RI, pode estar apontando para a possibilidade de utilização do índice enquanto indicador da presença de RI associada à $S M$, o que poderia vir a favorecer a definição da abordagem terapêutica e, conseqüentemente, o prognóstico dos indivíduos acometidos, mais especificamente os não diabéticos (5,37-40).

Para tanto, contudo, estudos adicionais ainda se fazem necessários, uma vez que nossos achados, tal como os de outros autores, estão atrelados às peculiaridades das populações envolvidas, às suas características genéticas e exposições ambientais específicas, que tanto influenciam a ocorrência da hipertensão arterial, das dislipidemias e demais alterações metabólicas.

\section{AGRADECIMENTOS}

Ao Prof. Dr. Fernando Martins Carvalho, da Faculdade de Medicina da Bahia (UFBA), pela extensiva e cuidadosa revisão deste artigo, sobretudo quanto aos aspectos estatístico-metodológicos; ao Prof. Dr. José Tavares-Neto, Diretor da Faculdade de Medicina da Bahia, por disponibilizar a infra-estrutura local do Projeto Cavunge.

\section{REFERÊNCIAS}

1. Hopkins PN, Williams RR. A survey of 246 suggested coronary risk factors. Atherosclerosis 1981;40:1-52.

2. Reaven GM. Banting lecture 1988: role of insulin resistance in human disease. Diabetes 1988;37:1595-607.

3. Kaplan NM. The delay quartet: upper-body obesity, glucose intolerance, hypertriglyceridemia and hypertension. Arch Intern Med 1989;149:1514-20.

4. National Institutes of Health. Executive summary of the third report of the national cholesterol education program (NCEP) expert panel on detection, evaluation and treatment of high blood cholesterol in adults (adult treatment panel III). JAMA 2001;285:2486-97.

5. Stern SE, Williams K, Ferrannini E, DeFronzo RA, Bogardus C, Stern MP. Identification of individuals with insulin resistance using routine clinical measurements. Diabetes 2005;54:333-9.

6. American College of Endocrinology. Executive summary of the Position statement on the insulin resistance syndrome. Endocr Pract 2003;9(3):237-9.

7. Matthews DR, Hosker JP, Rudenski AS, Naylor BA, Treacher DF, Turner RC. Homeostasis model assessment: insulin resistance and beta-cell function from fasting plasma glucose and insulin concentrations in man. Diabetologia 1985;28:412-9.

8. Wallace TM, Matthews DR. The assessment of insulin resistance in man. Diabet Med 2002;19:527-34.

9. Monzillo LU, Handy O. Evaluation of insulin sensitivity in clinical practice and in research settings. Nutr Rev 2003;61(12):397-412.

10. Wallace TM, Levy J, Matthews DR. Use and abuse of HOMA modeling. Diabetes Care 2004;27(6):1487-95.

11. Oliveira EP, Souza MLA, Lima MDA. Índice HOMA (homeostasis model assessment) na prática clínica: uma revisão. J Bras Patol Med Lab 2005;41(4):237-43.
12. Oliveira EP, Souza MLA, Lima MDA. Prevalência de síndrome metabólica em uma área rural do semi-árido baiano. Arq Bras Endocrinol Metab 2006;50(3):456-65.

13. Karam JH, Forsham PH. Pancreatic hormones \& diabetes mellitus. In: Greenspan FS, Baxter JD (eds). Basic \& Clinical Endocrinology. $4^{\text {th }}$ ed. USA: Appleton \& Lange, 1994. pp. 571-634.

14. Balkau B, Charles MA. Comment on the provisional report from the WHO consultation: European Group for the Study of Insulin Resistance (EGIR). Diabet Med 1999;16(5):442-3.

15. Grundy SM. Metabolic syndrome: part I. Endocrinol Metab Clin North Am 2004;33:IX-XI.

16. Reaven $\mathrm{G}$. The metabolic syndrome or the insulin resistance syndrome? Different names, different concepts and different goals. Endocrinol Metab Clin North Am 2004;33:283-303.

17. Ford ES, Giles WH. The comparison of the prevalence of the metabolic syndrome using two proposed definitions. Diabetes Care 2003;26:575-81.

18. Meigs JB, Wilson PWF, Nathan DM, D'Agostino RB, Williams K, Haffner SM. Prevalence and Characteristics of the metabolic syndrome in the San Antonio Heart and Framingham offspring studies. Diabetes 2003;52:2160-7.

19. Resnick HE, Jones K, Ruotolo G, Jain AK, Henderson J, Lu W, et al. Insulin resistance, the metabolic syndrome and risk of incident cardiovascular disease in nondiabetic American Indians. Diabetes Care 2003;26:861-7.

20. Saely CH, Aczel S, Marte T, Langer P, Hoefle G, Drexel H. The metabolic syndrome, insulin resistance and cardiovascular risk in diabetic and nondiabetic patients. J Clin Endocrinol Metab 2005;90(10):5698-703.

21. Tipene-Leach D, Pahau H, Joseph N, Coppell K, McAuley K, Booker $\mathrm{C}$, et al. Insulin resistance in a rural Maori community. N Z Med J 2004;117(1207):U1208.

22. McAuley KA, Williams SM, Mann J, Walker RJ, Lewis-Barned $\mathrm{NJ}$, Temple LA, et al. Diagnosing insulin resistance in the general population. Diabetes Care 2001;24:460-4.

23. Bustos $P$, Amigo $H$, Arteaga $A$, Acosta AM, Rona R. Factores de riesgo de enfermedad cardiovascular en adultos jóvenes. Rev Méd Chile 2003;131:973-80.

24. Jaber LA, Brown MB, Hammad A, Zhu Q, Herman WH. The prevalence of the metabolic syndrome among Arab Americans. Diabetes Care 2004;27(1):234-8.

25. Bergman RN, Zaccaro DJ, Watanabe RM, Haffner SM, Saad MF, Morris JM, et al. Minimal model-based insulin sensitivity has greater heritability and a different genetic basis than the homeostasis model assessment or fast insulin. Diabetes 2003;52:2168-74.

26. Gokcel A, Baltali M, Tarim E, Bagis T, Gumurdulu Y, Karakose $\mathrm{H}$, et al. Detection of insulin resistance in Turkish adult: a hospital-based study. Diabetes Obes Metab 2003;5:126-30.

27. Bravata DM, Wells CK, Concato J, Kernan WN, Brass LM, Gulanski BI. Two measures of insulin sensitivity provided similar information in a U.S. population. J Clin Epidemiol 2004;57:1214-7.

28. Cameron AJ, Shaw JE, Zimmet PZ. The metabolic syndrome: prevalence in worldwide populations. Endocrinol Metab Clin North Am 2004;33:351-75.

29. Reaven GM. Insulin resistance/compensatory hyperinsulinemia, essential hypertension and cardiovascular disease. J Clin Endocrinol Metab 2003;88(6):2399-403.

30. Assmann G, Nofer JR, Schulte H. Cardiovascular risk assessment in metabolic syndrome: view from PROCAM. Endocrinol Metab Clin North Am 2004;33:377-92.

31. Jeffs JAR, Godsland IF, Johnston DG. Less than $50 \%$ of variation in HDL cholesterol between and within individuals is explained by estabilished predictors. Atherosclerosis 2005;184(1):178-87.

32. Park YW, Zhu S, Palaniappan L Heshka S, Carnethon MR, Heymsfield SB. The metabolic syndrome: prevalence and associated risk factor findings in the US population from the third national health and nutrition examination survey, 19881994. Arch Intern Med 2003;163:427-36.

33. Natali A, Ferrannini E. Hypertension, insulin resistance and the metabolic syndrome. Endocrinol Metab Clin North Am 2004;33:417-29. 
34. Mohteshamzadeh M, Wilkinson $\mathrm{R}$, Thomas SHL. Insulin resistance in men with treated hypertension at increased risk for cardiovascular disease: results of a 3-year study. Am J Hypertens 2005; 18:452-6.

35. Taniguchi A, Nakai Y, Sakai M, Yoshii S, Hamanaka D, Hatae $Y$, et al. Relationship of regional adiposity to insulin resistance and serum triglyceride levels in non-obese Japanese type 2 diabetic patients. Metabolism 2002;51(5):544-8.

36. Pacini G, Mari A. Methods for clinical assessment of insulin sensitivity and beta-cell function. Best Pract Res Clin Endocrinol Metab 2003; 17:305-22.

37. Stumvoll $M$, Häring $H$. Insulin resistance and insulin sensitizers. Horm Res 2001;55(suppl 2):3-13.

38. Füllert S, Schneider F, Haak E, Rau H, Badenhoop K, Lubben $\mathrm{G}$, et al. Effects of pioglitazone in nondiabetic patients with arterial hypertension: a double-blind, placebo-controlled study. J Clin Endocrinol Metab 2002;87(12):5503-6.
39. Goldstein BJ. Insulin resistance as the core defect in type 2 diabetes mellitus. Am J Cardiol 2002;90(5A):3G-10G.

40. Orchard TJ, Temprosa M, Goldberg R, Haffner S, Ratner R, Marcovina $S$, et al. The effect of metformin and intensive lifestyle intervention on the metabolic syndrome: The Diabetes Prevention Program Randomized Trial. Ann Intern Med 2005;142:611-9.

\section{Endereço para correspondência:}

Ernesto P. de Oliveira

Faculdade de Farmácia da UFBA

Rua Barão de Geremoabo s/n

40170-290 Salvador, BA

E-mail: epo@ufba.br 